BULLETIN Bulletin hispanique

HispaniQuE Université Michel de Montaigne Bordeaux

$116-2 \mid 2014$

Référentialité/autoréférentialité dans le roman espagnol contemporain : bilan et perspectives

\title{
Médiations du réel et pulsion scopique chez Juan José Millás
}

Jean-François Carcelen

\section{(2) OpenEdition}

Journals

Édition électronique

URL : http://journals.openedition.org/bulletinhispanique/3503

DOI : 10.4000/bulletinhispanique.3503

ISBN : 979-10-300-0156-3

ISSN : $1775-3821$

Éditeur

Presses universitaires de Bordeaux

Édition imprimée

Date de publication : 1 décembre 2014

Pagination : 685-699

ISBN : 978-2-86781-963-6

ISSN : 0007-4640

Référence électronique

Jean-François Carcelen, « Médiations du réel et pulsion scopique chez Juan José Millás », Bulletin hispanique [En ligne], 116-2 | 2014, mis en ligne le 01 décembre 2017, consulté le 19 avril 2019. URL : http://journals.openedition.org/bulletinhispanique/3503; DOI : 10.4000/bulletinhispanique.3503 


\title{
Médiations du réel et pulsion scopique chez Juan José Millás
}

\author{
Jean-François Carcelen \\ Université Paul Valéry Montpellier 3
}

Juan José Millás ne cesse d'expérimenter de nouvelles modalités afin de rendre sensible le réel, notamment par une présence de plus en plus forte de limage dans son écriture. À travers le fotocuento, il montre la nécessité de l'écrit pour dépasser le simple analogon du réel, et dans son roman $\mathrm{El}$ mundo, il fait de la médiation par l'image et les effets-image, la modalité autoréférentielle par laquelle s'exerce la référence. Chacune des deux tensions, référence et autoréférence, semble ainsi être ce par quoi l'autre advient.

Mots-clés : Juan José Millás, référence, autoréférence, image, métatextualité

Juan José Millás experimenta sin cesar nuevas modalidades para hacer sensible la realidad, en particular a través de una presencia cada vez más fuerte de la imagen en su escritura. A través del fotocuento, muestra el autor la necesidad de la escritura para rebasar el mero analogon de lo real. En su novela El mundo, la mediación por la imagen y los efectos-imagen se convierte en la modalidad autorrefencial a través de la cual se ejerce la referencia. Cada una de las dos tensiones, referencia y autorreferencia, viene a ser asi lo que permite la existencia de la otra.

Palabras clave: Juan José Millás, referencia, autorreferencia, imagen, metanovela

Juan José Millás does not stop examining and experimenting new ways of making reality discernible, in particular by including a broader presence of the image in his writing. Through the fotocuento, he shows the necessity of writing in order to go beyond the simple analogon of reality, and in his novel $\mathrm{El}$ mundo, he mediates through the image and the image effects, the self-referential mode by which he exerts the reference. Each of these two tensions, reference and self-reference, therefore seems to be what through which the other occurs.

Keywords: Juan José Millás, referential, self-referential, image. 
$\mathrm{E}$ n 1991, lors du colloque Référence et autoréférence dans le roman espagnol contemporain, encore fortement imprégnés par les théories dominantes selon lesquelles le référent n'était pas soluble dans l'écriture et qu'en conséquence le texte ne pouvait que construire son propre référent, nous nous demandions si le texte littéraire était apte à référer, à dire le monde. Il était donc inutile de vouloir chercher, derrière l'opacité des signes, la transparence du monde, et il fallait envisager dans sa clôture un texte qui parlait avant tout de lui-même. Il nous fallait cependant affronter une contradiction apparente puisque la lecture et l'étude des romans nous montraient que le réel n'était jamais bien loin, qu'on en percevait ses traces, sa dynamique, sa pulsion. Il fallait donc avoir une approche beaucoup plus nuancée et affirmer, après d'autres, que référence et auto-référence n'étaient nullement incompatibles, ni exclusives l'une de l'autre. Ce sont bien les deux pôles d'une même tension, ce que la formule aussi précise que définitive de Claude Abastado avait bien résumé : " en un discours unique (...) le texte dit le monde et se dit lui-même ${ }^{1}$ ", ou encore, en des termes empruntés à Geneviève Champeau : " la fiction fait ce qu'elle dit et finit par être ce dont elle parle ".

\section{Constat Circonstanciel : QUAND LE RÉEl Fait Retour}

Ces dernières années ont supposé une exacerbation des deux tensions : jamais la littérature n'a fait autant retour sur elle-même, au point, dans certains cas, de n'être constituée que par des textes faits de prélèvements d'autres textes, et non plus de simples citations ou allusions intertextuelles. Et dans le même temps, voire dans le même espace textuel, jamais elle n'a été taraudée à ce point par la question du réel. Il semble bien que la tendance à vouloir signifier le monde se soit accentuée et que les écrivains n'aient eu de cesse de renouveler les approches de leur rapport au réel. En Espagne, le roman policier des premières années de la transition, le « realismo sucio " des années 90, le roman de la mémoire et sa forte tension documentaire en sont quelques exemples. Parmi les modalités les plus récentes de la référence, on peut ainsi observer une pénétration chaque fois plus grande du discours argumentatif dans le discours fictionnel, ou encore du document dans des romans "scénographiés " dont l'approche doit souvent se faire en termes de "montages ».

Étrange paradoxe qui veut qu'à une époque où tout semble scénarisé, où l'on fait fiction de tout bois, certains aient pu observer dans le même temps la fin de l'hégémonie des discours de fiction. Au fond, quelle que soit la façon de l'envisager, il semble bien que la littérature ne puisse échapper à la question de ses rapports au réel².

1. Claude Abastado, "La glace sans tain ", Littérature, n² 27, 1977. Métalangages, p. 55.

2. Geneviève Champeau l'avait très bien saisi et exprimé dans la conclusion de son avantpropos aux actes du colloque de 1991 : "S'il ne réfère ni n’autoréfere au sens strict, le roman ne renonce ni à signifier le monde ni à parler de lui-même, mais il le fait à sa manière qui consiste à faire entendre plus qu'il ne dit, par la logique des signifiants. ", Geneviève Champeau, "Avantpropos ", Référence et autoréférence dans le roman espagnol contemporain, Bordeaux, Maison des 
Dans un monde où domine la "culture écranique ", ou le réel est de plus en plus une forme médiatisée, un réel métamédiatique, selon David Foster Wallace, plus qu'un réel sensible, un réel auquel on accède par des intermédiaires, au point même que dans des cas extrêmes - et sans doute pathologiques comme dans ces expériences limites de vies se déroulant dans le virtuel pur (Second life etc.) - le média est le réel, il est assez logique de concevoir que les deux tensions (référentielle et autoréférentielle) se superposent, se confondent dans un même objectif : "(d)écrire » le réel. Peut-on alors postuler que nous sommes dans un au-delà de la complémentarité et que chacune des deux tensions est ce par quoi l'autre advient ? L'écrivain est en effet obligé de se mouvoir entre deux constants rappels à l'ordre: d'une part le monde qui sans cesse vient "faire pression $^{4}$ ", d'autre part ce miroir en permanence sous les yeux, ce langage qui se rappelle sans cesse à lui, cette matière qu'il a en charge de modeler. Comme le dit Juan José Millás : "Las palabras son las intermediarias entre la realidady nosotros.»

C'est donc à partir de ce postulat que je voudrais proposer ici une réflexion sur quelques-unes des modalités référentielles mises en œuvre par Millás dans la diversité de ses expériences littéraires. Expériences qui sont autant de réflexions sur les outils discursifs lui permettant, sinon de faire advenir le référent dans le texte littéraire, du moins de le rendre sensible au lecteur.

On a beaucoup dit que Juan José Millás était un écrivain entièrement tourné vers ses obsessions personnelles ${ }^{5}$, que ses columnas étaient écrites depuis une distance littéraire qui les éloignait de la chronique journalistique, ou encore que ses romans étaient marqués par une forte tension métatextuelle. Cependant, son parcours est assez emblématique de la question qui nous occupe. Certes, on fait écrivain plus "réaliste ", mais à sa façon, Juan José Millás est un auteur profondément concerné par le monde, comme il a pu le démontrer tout au long de ces vingt-trois dernières années dans son écriture de presse.

Dans ses réalisations les plus récentes, Juan José Millás semble avoir ajouté un nouvel item à sa panoplie d'obsessions, que nous pourrions nommer l'obsession scopique. Fasciné par l'image et le regard, sans doute depuis ses premiers textes, il semble avoir accepté que dans une "culture écranique ", plus rien ne puisse se définir autrement qu'en termes de cadrage ou de point de vue. Ce faisant, il n'oblitère pas pour autant la possibilité de signifier le monde, au contraire, il s'engage dans un travail multiforme ${ }^{6}$ (radio, télé, internet, Articuento, roman)

Pays Ibériques, 1994, p. 10.

3. Voir Gilles Lipovetsky et Jean Serroy, L'écran global : Culture-médias et cinéma à l'âge hypermoderne, Paris, Le Seuil, 2007.

4. Dominique Viart et Bruno Vercier, La Littérature française au présent : héritage et mutations de la modernité, Paris, Bordas, 2008, p. 11.

5. Je renvoie aux travaux de Gonzalo Sobejano, Juan José Millás, fabulador de la extrañeza (Madrid, Alfaguara, 1995) ou, plus récemment, à l'article d'Élide Pitarello : "Las metamorfosis de Juan José Millás », dans Geneviève Champeau et alii, Nuevos derroteros de la narrativa española actual, Saragosse, PUZ, 2011.

6. Après son incursion réussie dans la presse (où récemment encore, en 2013, il a prolongé 
dont le propos central est bien de questionner le réel et ses représentations, pour en saisir non l'écume, mais toute la complexité.

L'expérience de la columna l'amène donc à envisager l'écriture dans une double tension intrinsèque à ce type d'expérience si l'on en croit Marie-Eve Thérenty : «En tant que journaliste, il doit s'affronter aux problèmes que pose la retranscription du réel. En tant qu'écrivain, il est tenté par la mise en fiction de ce réel ${ }^{7}$ ". Car, comme il l'avoue lui-même, cette écriture est absolument solidaire de celle qui régit le reste de sa production en particulier ses romans : "En el periodismo puede haber un elemento de regeneración de la novela. Cuando se cruzan esos dos géneros, periodismo y novela, resulta que se regeneran los dos. "

On peut donc avancer sans risques que le projet d'écriture de Millás vise à rendre compte du monde à travers une expérience, la sienne, qui s'actualise dans un discours dont les contours semblent sans cesse questionnés. Ainsi, la columna ou plutôt ce que l'on appelle aujourd'hui articuento, selon le néologisme créé par l'auteur lui-même pour un genre dont lui seul aurait les clés, est-elle une des modalités privilégiées pour déconstruire - et en même temps, paradoxalement, construire - un réel qu'il ne peut approcher qu'à travers un langage qui est lui aussi à déconstruire, à réfléchir.

\section{ICONe ET GRAPHie : LeS Fotocuentos de JuAn José Millás}

Après el articuento, et sans doute directement lié à lui, Juan José Millás " invente " un autre " genre littéraire bref " ${ }^{8}$ qui lui permet d'affiner son regard sur le monde, de proposer une façon singulière d'envisager le réel à partir de son analogon, la photographie. Dans la section intitulée "La imagen ", publiée dans le supplément El País semanal en 2004, 2005 et 2006, et prolongée jusqu'en 2013, Juan José Millás inaugure dans son écriture ce que l'on pourrait appeler par analogie avec "articuento ", le "fotocuento". Il s'agit d'un commentaire de photo, d'une expansion de la légende photographique, transformée ainsi en objet littéraire, comme l'a très bien analysé Âlvaro Romero Bernal : «Millás consigue crear otro género breve nuevo, tras vivificar la columna hasta el punto de llevarla a extremos literarios, convierte el pie de página en auténtico género literario. Trabajando en profundidad el paradigma archivistico, es la versión millasiana de la literatura documental ${ }^{9} "$.

l'expérience du commentaire photographique commencée en 2004 dans la section «La imagen» de El País semanal), Millás s'est essayé à la radio (émission "La ventana», sur la Cadena Ser), etc.

7. Marie-Eve Thérenty, "Contagions : fiction et fictionalisation dans le journal autour de 1830 ", Colloque en ligne Frontières de la fiction, 1999, http://www.fabula.org/forum/ colloque99/214.php.

8. Álvaro Romero Bernal, «Cuando una imagen no vale más que mil palabras: el paradigma de las 'fotos resucitadas' por Juan José Millás en El País Semanal (año 2010) ", Actas - II Congreso Internacional Latina de Comunicación Social - Universidad La Laguna, diciembre de 2010, Disponible sur http://www.revistalatinacs.org/10SLCS/actas_2010/Romero.pdf, consulté le 10 juillet 2012.

9. Ibid. 
Cet espace d'écriture correspond à une mise en récit d'un fragment " emprunté " au réel par le biais de la photographie mais qui, sans l'apport de l'écrivain, resterait le plus souvent une sorte d'assertion, voire une simple illustration, sur laquelle le regard du lecteur, saturé d'images, hésite un instant et glisse le plus souvent. Même lorsque la photo est d'une densité émotionnelle extrême, le fameux " choc des photos ", Juan José Millás dépasse par son commentaire la sidération que l'image est capable d'engendrer et engage un dialogue entre dénotation et connotation à travers lequel il parvient à faire émerger le réel dans toute sa complexité. C'est ainsi un singulier travail sur l'Archive photographique, que l'écrivain nous propose, en même temps qu'une réflexion sur la difficulté et la nécessité de rendre compte du réel. Comme l'a très bien montré Georges Didi-Hubermann, l'image c'est du « temps qui nous regarde ». Juan José Millás saisit parfaitement ce temps qui lui fait face et lui enjoint d'une certaine façon de « re-monter » l'histoire :

Mettre le temps au centre de toute pensée de l'image : nous sommes devant l'image comme devant du temps - car dans l'image c'est bien du temps qui nous regarde. [...] [les images] sont des montages de temporalités différentes, des symptômes déchirant le cours normal des choses. Quand l'image survient, l'histoire se "démonte ", dans tous les sens du mot. Mais alors, le temps se montre, il s'ouvre dans toute sa complexité, dans son montage de rythmes hétérogènes formant anachronismes ${ }^{10}$.

Je voudrais faire une brève analyse de cette modalité d'écriture millassienne à partir d'un exemple précis, le fotocuento intitulé "Confusion y estupor ${ }^{11}$ " et publié pour la première fois le 4 août 2005 dans le supplément El País semanal.

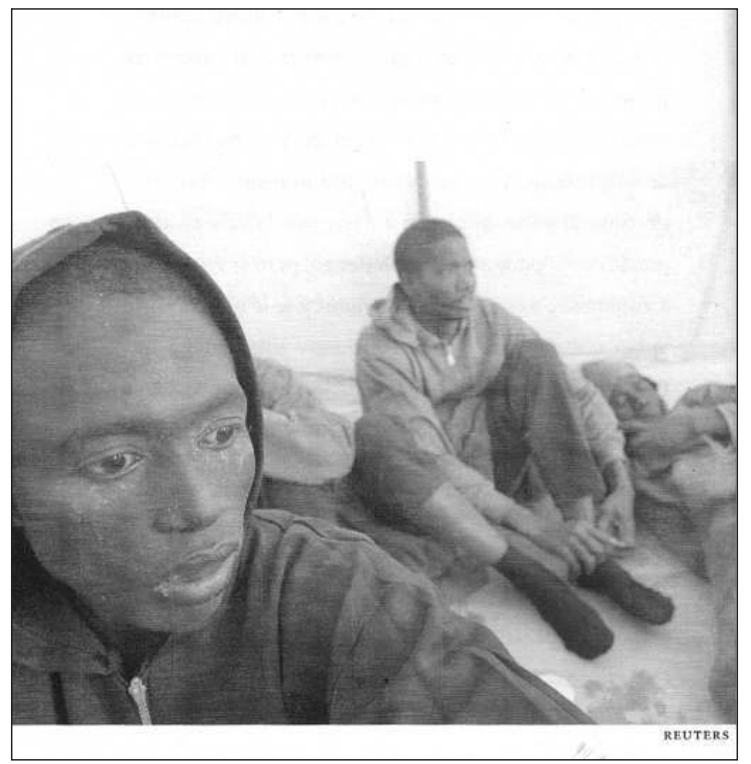

Confusión y estupor.

Agence Reuteurs, reprise par Juan José Millas dans... cf. note 11

10. Georges Didi-Huberman, Devant le temps, Paris, Minuit, 2010.

11. Juan José Millás, El ojo de la cerradura, Barcelone, ediciones Península, 2006, p. 26. 
Cuando un cuerpo se encuentra expuesto al frio, el organismo estrecha los vasos sanguineos menos importantes para dirigir mayor cantidad de sangre a los centros vitales. Las partes que dejan de recibir sangre (dedos, orejas, labios, párpados...) se enfrian más rápidamente, adquiriendo una rigidez como la que se aprecia en el rostro del hombre de la foto. Se ha quedado, literalmente, de piedra. Colaboran a subrayar esa impresión los restos de sal marina que dibujan vetas como de mármol sobre su cara. La cabeza, por su forma, pero también por la gran cantidad de piel empleada en el tapizado de la calavera, es por donde más calor se pierde. De ahi el gorro de los esquiadores y los alpinistas, o el sombrero de los calvos.

De entre los males causados por la exposición al frio, el más común es la hipotermia, que consiste en una temperatura corporal anormalmente baja. De no actuar rápidamente, proporcionando a la víctima alimentos y mantas, el frio puede rebasar la frontera de la piel y afectar a los órganos internos. Cuando rompe las defensas del cerebro y entra como una aguja helada en sus zonas sensibles, produce estados de delirio o confusión, también de estupor. Por ello, aunque la persona permanezca consciente, puede dar muestras de desorientación. Si tiene sitio para caminar, lo hará de forma errática, con la mirada perdida, como si buscara un lugar inexistente en la dimensión en la que se mueve. Tampoco es raro que confunda la actualidad con el pasado o que crea que se encuentra en un lugar distinto del que está. La disminución de la actividad intelectual suele ir acompañada de cierto aire de asombro como el que percibimos también en este hombre, que acaba de ser recogido de una embarcación en la que viajaban más de cuarenta personas, 13 de ellas muertas.

La hipotermia entra en el cuerpo de puntillas. Ataca, por lo general, a personas que no se pueden mover y con las ropas mojadas. Durante unos instantes, no sabes si el frío está en tu camisa o en tu piel. Cuando adviertes que está en tu piel, seguramente ya ha dañado a varios conjuntos de células. En palabras del médico que se hizo cargo de los vivos y los muertos de esta patera, que llevaba dos o tres dias en alta mar, sin alimentos, sin agua y con temperaturas de entre siete y diez grados, las víctimas "comienzan por sentir un frio muy intenso, tiritan primero $y$ luego sufren convulsiones, la sangre comienza a bajar de 35 grados y los músculos comienzan a agarrotarse hasta que llega una parada cardiorrespiratoria".

La exposición continuada a este tipo de noticias provoca a la larga una insensibilidad social semejante al estupor. Vemos la fotografía en el periódico, leemos el titular, y pasamos de página. El titular de esta noticia aseguraba que los que se salvaron lo hicieron gracias al calor que se daban unos a otros, pues iban abrazados. Dentro de los próximos años, la única posibilidad de que se salven ellos y nosotros es que nos abracemos. Lo veríamos con claridad de no ser por el estado de delirio, confusión y estupor en el que hemos caído.

Cet article fait dialoguer deux documents émanant de deux auteurs différents : un document photographique que l'on doit analyser en tant que tel et un texte qui à la lecture se révèle lui aussi très documentaire, dans une sorte de froideur scientifique. Seuls quelques points de contact cousent l'un à l'autre, mais ils suffisent à montrer leur indissociabilité. C'est celle-ci qui en fait un discours unique.

La photo permet de saisir la sidération évoquée précédemment, le spectateur est sidéré face à l'épaisseur de ce réel qui fait brutalement irruption dans son champ de vision. Dans le regard de ce rescapé, semble encore poindre la mort, celle qu'il a frôlée, celle qu'il a côtoyée. Le texte de Millás se dérobe dans un premier temps à l'eckphrasis, voire au commentaire. À partir d'un présent de généralité, le narrateur, tel un médecin légiste, fait un détour par la description clinique et glaciale, presque déshumanisée, des effets du froid et de l'humidité sur les corps. Ce procédé, loin de nous éloigner de la réalité brutale, et immédiatement poignante, qui émane de la photo, nous ramène au cœur- 
même de celle-ci, car la violence de la scène photographiée est ainsi exacerbée par le contraste entre cette écriture "blanche " et la tension dramatique perceptible dans ce visage pétrifié.

Juan José Millás glisse peu à peu de la froideur initiale à l'émotion dans une conclusion où le pathos prend le relais de l'objectivité. Il réussit ainsi, en mettant des mots sur un regard et un regard sur des mots, à provoquer un " choc thermique " qui nous fait mesurer toute l'ampleur du réel. L'homme photographié cesse alors d'être un simple rescapé pour devenir le survivant d'une humanité naufragée dont nous faisons tous partie. Millás s'attache, en effet, à s'impliquer et à impliquer le lecteur dans l'événement : "Dentro de los próximos años, la única posibilidad de que se salven ellos y nosotros es que nos abracemos. Lo veríamos con claridad de no ser por el estado de delirio, confusión y estupor en el que hemos caído."

C'est bien dans cette dialectique entre le document et le texte, l'image et le langage, que Millás déploie son écriture. Il y a chez lui, depuis ses premiers romans, une volonté de déconstruire le réel et une certitude : celui-ci ne se donne pas à voir directement, dans une sorte d'immanence qui le réduit à une apparence, il nécessite une obliquité, un ou des points de vue, un cadrage. Récemment encore dans un article intitulé "La necesidad del encuadre ", appartenant à la dernière livraison pour la section "La imagen ", il prenait la question du cadrage comme sujet, dans une réflexion metaréférentielle (metaperiodistica selon Romero Bernal) conclue par cette phrase : "La necesidad del encuadre, incluso del recuadro, para entender la realidad ${ }^{12}$."

La plupart du temps, l'image photographique de presse est essentiellement considérée dans l'article qu'elle accompagne ou illustre, dans la dénotation. Pourtant, elle est livrée au regard du lecteur dans toute sa complexité, et selon ce qu'elle montre, elle provoque une onde de choc, une interrogation, un étonnement, quelque chose qui n'est pas de l'ordre de l'explicite, de la dénotation, mais de l'ordre de l'implicite et de la connotation. C'est précisément cette onde de choc, cet espace vide, ou plutôt absent, que Juan José Millás cherche à traquer. Cet espace, c'est celui de la "mirada ": "Todo el mundo tiene una cámara de fotos, pero no todo el mundo tiene mirada $»^{13}$, dit-il dans son prologue à El ojo de la cerradura.

Depuis un double décalage par rapport à l'événement, spatial et temporel, Millás « rescata $^{14}$ » des fragments de temps tombés dans l'oubli, mais nécessaires. Face à la sursaturation iconique, il faut en effet le détour par l'écrit, qui agit comme une sorte de pause réflexive, pour pouvoir retrouver toute la force d'un réel que son analogon photographique ne semble plus à même de restituer.

Parallèlement à son écriture de presse, Juan José Millás continue sa trajectoire romanesque. Cette coïncidence temporelle de deux écritures de nature différente n'est pas sans conséquences puisque les influences réciproques

12. Juan José Millás, «La necesidad del encuadre », dans El País semanal, 13 juin 2013.

13. El ojo de la cerradura, p. 9

14. Le terme est utilisé avec bonheur par Romero Bernal dans l'article cité antérieurement. 
sont manifestes. Comme le souligne l'auteur lui-même, les deux écritures se nourrissent mutuellement : "En el periodismo puede haber un elemento de regeneración de la novela. Cuando se cruzan esos dos géneros, periodismo y novela, resulta que se regeneran los dos. " Ainsi, lorsque l'image photographique devient l'objet d'une réflexion centrée, comme nous l'avons dit, sur le réel, sa représentation iconique et son herméneutique textuelle, Millás construit un roman traversé par l'obsession de l'image, animé d'une pulsion scopique en rapport direct avec les interrogations qu'il affronte dans les fotocuentos de la section Imagen. Ce qui apparait très clairement dans une question que le narrateur de $E l$ mundo, en pleine lecture d'un roman, pose ouvertement, presque "à la cantonade " : ¿ ¿ Cómo era posible que, habiendo sólo letras, yo viera solamente imágenes ?15 " (182). Je voudrais ici montrer comment la médiation à l'oeuvre dans El mundo, principalement à travers l'image et les effets-image, est la modalité autoréférentielle par laquelle s'exerce la référence.

\section{D'ÉCRIRE LE RÉEL : LE MONDE DEPUIS EL MUNDO}

Dans El mundo, prix Planeta 2007, Juan José Millás fait un retour sur luimême et sur sa trajectoire littéraire. Si El mundo est considéré comme un roman par l'ensemble de la critique, il pose cependant quelques problèmes de classement générique. Il s'agit d'un récit autobiographique articulé autour de quatre moments essentiels de la formation de Juanjo Millás ${ }^{16}$ (enfance et adolescence principalement), un parcours destiné à expliquer l'adulte et l'écrivain qu'il est devenu, bien que finalement cette tentative soit une aporie puisqu'au bout du compte le narrateur dans un éclair de lucidité affirme : «... lo cierto es que resulta imposible entender lo que soy a partir de lo que fui " (El mundo, 203). Juan José Millás expose ainsi dans cette autobiographie imprégnée d'autocritique génétique, les différents sédiments que certaines expériences clés de sa vie ont laissés dans son œuvre. Roman de formation tout autant qu'introspection, autofiction peut-être, El mundo se caractérise donc par une hétérogénéité générique au sein de laquelle les données factuelles se mêlent à de nombreux passages digressifs, autoréflexifs, voire métatextuels, et de multiples récits enchâssés. L'auteur y fait aussi une sorte "d'album familial ", à partir d'images mentales, de réminiscences, et finit par nous offrir un texte au pacte de lecture ambigu. L'épitexte a beau insister sur la dimension autobiographique, Juan José Millás oscille dans ce roman en permanence entre déni et délit de fiction.

15. Juan José Millás, El mundo, Barcelone, Editorial Planeta, 2007, p. 182. Dorénavant, les citations extraites du roman seront directement signalées dans le corps de l'article par El mundo suivi de la page concernée.

16. Si l'utilisation du diminutif du prénom peut objectivement signifier une sorte de mise à distance de l'auteur par rapport au protagoniste narrateur, et donc venir nuancer le pacte autobiographique, la plupart de ses lecteurs savent que c'est ainsi que l'on appelle Millás dans « la vie courante». 
El mundo est un roman écrit de l'intérieur de la réalité qu'il cherche à déconstruire, du cœur même de cette réalité, en l'occurrence celle d'une époque franquiste où dominent la peur et la frustration. Cette situation ne pouvait engendrer chez l'enfant, puis l'adolescent qu'était alors Millás, qu'une lecture faussée d'un réel qui s'offrait à lui comme un artifice ou un artefact, comme une construction morale et idéologique. D'un point de vue référentiel, l'essentiel du roman a donc pour cadre une dictature encore bien en place, les ténèbres du franquisme vues à travers une enfance en clair-obscur ${ }^{17}$. La tension autobiographique qui le régit est en même temps le garant d'un temps et d'un espace attestés dans l'histoire, du moins dans celle de Juan José Millás.

Les mécanismes référentiels dans ce roman vont bien au-delà de la présence - finalement bien sporadique - d'ancrages toponymiques ou chrononymiques. La réalité espagnole convoquée ici est beaucoup plus métaphorisée ou insinuée que décrite et pourtant, dans la singularité du prisme par lequel elle est saisie, le lecteur a l'impression d'assister à une véritable autopsie sociale. Parfois un seul petit point d'ancrage référentiel suffit à baliser l'inscription dans l'Histoire et permet à la fiction de se déployer avec une forte charge de vérité. Une scène traduit très bien cela, dans laquelle le narrateur se rappelle une rencontre avec María José : "La última vez que la vi durante aquel curso fue en el célebre recital de Raimon, en la Facultad de Económicas. " (El mundo, 144). Cette simple mention sert de cadre référentiel à un épisode où le narrateur exalte la résistance étudiante au franquisme, incarnée par María José, face à la brutalité des forces de l'ordre... et à sa propre lâcheté qui ne lui permet d'assister à la scène que caché derrière un arbre, depuis une passivité a posteriori coupable.

Dans cet exercice de rétrospection, ce qui résiste à la compréhension de l'enfant, et sans doute à celle de l'adulte qu'il est devenu, c'est l'opacité du monde, cet épais voile qui semble s'interposer entre le narrateur Juanjo Millás et un réel dont il a du mal à cerner les codes. Un voile qu'il cherche constamment à déchirer, par des stratégies d'évitement ou de contournement qui finissent par être de véritables stratégies de dévoilement, au premier rang desquels se trouve l'écriture.

Tras aquel "Tú no eres interesante (¿para mí?)", la opacidad se acentuó. Era opaco el patio del colegio; eran opacos los curas y los compañeros; opacos los libros de texto; opacos mis hermanos y los confesionarios y las absoluciones; opacas las misas; eran opacos Dios y el diablo y opacas las horas de la vigilia y el sueño; el frio era opaco y opacas las discusiones de mis padres; opacos los bultos de todos los pasillos y opacas las acelgas que se manifestaban cada noche en los opacos platos desportillados de la cena. Era opaco yo, entre las sábanas, y opacas las manos con

17. Le motif du clair-obscur est présent à plusieurs reprises dans le texte. Le narrateur en explicite lui même la valeur métaphorique : "Al poco llegó mi madre con una vela encendida que colocó sobre la mesilla de noche. Tras suspirar, se sentó al pie de la cama, en el lado contrario al mío. La escena adquirió unos tonos algo sombrios. La llama de la vela se reflejaba en el espejo de cuerpo central del armario del dormitorio. Todo el mundo tiene un espejo de referencia, un espejo que le gustaría atravesar para llegar al otro lado de la vida. El mío era éste. Quizá lo sea aún." (El mundo, 211) Ou encore : "...la escena, lúgubre por la naturaleza de la luz que la alumbraba, sucedía al otro lado del espejo, al otro lado de la vida" (212). 
las que me tapaba desesperadamente los oídos para no escuchar las peleas de los mayores. Eran opacas mis fantasias sexuales y opaco mi sexo. Eran también opacos los meses y los años, que pasaban unos detrás de otros, como la procesionaria. Era opaco el futuro. (El mundo, 177)

L'enjeu est bien là : traquer, cerner, affronter tout ce qui opacifie. Retrouver le point de vue, le bon angle, le cadrage depuis lequel percevoir, pénétrer la réalité qui se cache derrière la jungle des apparences. C'est donc autant sur le regard que sur l'objet regardé que porte la réflexion du narrateur. Apprendre à voir, voir pour écrire et écrire pour voir : une obsession que Millás semble décliner sans cesse, quelle que soit la modalité d'écriture choisie.

Rien d'étonnant alors à ce que se développe dans le texte toute une série de figures, de motifs, de procédés qui ressortissent à cette opacité qui fait écran, rien d'étonnant à ce que les verbes "ver", "mirar ", " observar ", " reparar " et d'autres synonymes viennent saturer le récit. Il s'agit de "ver la callelenseñar la calle/mirar». La calle étant le monde initial et initiatique du jeune Millás, une "calle-mundo" dont les contours délimitent des espaces autres, l'espace de l'Autre aussi, chargés de tous les fantasmes et de tous les possibles : " ... la calle de Canillas, por otro lado, era el límite de la realidad. Más allá se extendía una sucesión de vertederos y descampados amenazadores, una especie de nada sucia que flotaba hasta donde alcanzaba la vista "(El mundo, 24).

Lorsque Vitaminas, son ami d'enfance, par ailleurs l'un des protagonistes principaux du second roman de Juan José Millás, Visión del ahogado ${ }^{18}$, isolé du monde par une maladie qui l'empêche de s'éloigner de chez lui, l'entraîne vers le "sótano" de l'épicerie paternelle et lui dit ces quelques mots sibyllins : "vamos a ver la calle " (El mundo, 47), alors qu'ils y sont, c'est tout un programme sur la façon de voir le réel - métaphorisé par la calle - qui s’ouvre alors pour lui. Ce fenestron ("respiradero ») devient ainsi physiquement le cadre à travers lequel les deux enfants vont envisager la calle autrement et avoir accès à la réalité derrière les apparences. Je n'insiste pas sur le sens symbolique que prend le mot "respiradero » dans cette Espagne en permanence sous surveillance, rance et étouffante. "Ver la calle " demande un effort, c'est un véritable exercice physique. Il faut en effet attendre le moment adéquat, descendre à la cave par une trappe et une échelle presqu'à la verticale, monter sur une caisse en bois pour enfin accéder à une filtration du réel : " ... habia un respiradero situado al nivel de la calle, se colaba una porción de luz. El respiradero se encontraba cubierto por una reja metálica muy tupida, la mayor parte de cuyos agujeros estaban cegados por una suciedad de siglos. " (El mundo, 47).

Ce faisant, le monde paradoxalement se dé-voile et apparaît chargé d'une autre dimension : "Era mi calle, sí, pero observada desde aquel lugar y a ras del suelo poseía calidades hiperreales, o subreales, quizá oníricas. " (El mundo, 48).

La réalité prend ainsi de l'épaisseur comme le montre les multiples anaphores du verbe voir ou encore ce glissement de ver vers les verbes mirar, puis observar et enfin reparar, dans un processus qui n'est pas sans rappeler la mise au point d'une focale. Ce déplacement du regard donne un autre point de vue, un autre

18. Juan José Millás, Visión del ahogado, Madrid, Alfaguara, 1977. 
accès au réel dans lequel on voit plus de choses ou les choses plus nettement : "de este modo reparé en la fuente que habia al lado de la puerta de mi casa, a la que jamás habia prestado atención alguna" (El mundo, 49). Après une longue litanie de tout ce qu'il pouvait enfin voir à travers la grille du fenestron, le passage se conclut de façon plus qu'évidente, notamment à travers la surmotivation onomastique, par l'apparition de la jeune adolescente dont il est amoureux : "Volvi a ver a Luz» (El mundo, 50). Cette même Luz qui, quelques pages plus loin, écrit à la machine les yeux bandés, produisant une "escritura ciega " à laquelle aspire le jeune Millás en plein désir d'écriture (El mundo, 73). Ce qu’il voit depuis ce regard oblique, c'est donc la lumière.

yo estaba obligado a contar la historia del mundo, es decir, la historia de mi calle, pues comprendi en ese instante que mi calle era una imitación, un trasunto, una copia quizá una metáfora del mundo. Intui también que debería emplear, para sacarla adelante, un método de la familia de la escritura ciega, que era, paradójicamente, le escritura de Luz. (El mundo, 92)

Pour saisir le monde (la Calle) il faut donc un point de vue assumé par un sujet narrant. Au contraire de cette écriture "blanche " qui caractérise les notes que remet Vitaminas à son père, soi-disant agent d'Interpol chargé de contrôler la zone, en fait délateur franquiste. Ces notes, purement factuelles, documentaires, vidées de tout point de vue, ne disent en fait rien du réel qu'elles croient consigner : "era preciso como un bisturí (eléctrico) y neutro como un atestado policial» (El mundo, 167).

Dans cette Espagne, le visible n'est donc qu'un trompe-l'œil. Comme dans cette image des deux adolescents -Juanjo et Luz- assis côte à côte dans une salle de classe, et dont les corps sont clivés : le haut (représentation du corps social, celui que l'on voit) est entièrement tourné vers l'apparence, l'assujettissement, le bas est le corps intime, celui qui sous le bureau s'essaie à la transgression. Les deux fonctionnent sur un mode différent et renvoient d'une façon oblique à la réalité espagnole sous le franquisme, dans ses conséquences traumatiques sur l'individu, celles que El mundo expose de la façon suivante : "comprendí oscuramente que la realidad estaba dividida en dos mitades (una de ellas invisible) que pese a ser complementarias, estaban condenadas a no encontrarse" (El mundo, 192).

\section{De l'ÉCRAN obstaCle À L'ÉCRAN RÉCEPTACle}

Si dans cet exercice, qui au fond s'apparente à une herméneutique du monde, le point de vue est essentiel, à l'autre bout du regard, il est aussi indissociable du cadrage indispensable à la compréhension du réel ${ }^{19}$. Si l'on excepte l'image iconique, totalement absente du roman, El mundo est saturé par l'image sous toutes ces formes : mentale (l'un des verbes structurant le récit est " recuerdo »), onirique, mnémonique, métaphorique. Millás met en place des procédés destinés à créer des effets spectaculaires en multipliant les effets de cadre et les effets d'écran.

19. Voir supra note 12. 
Le narrateur se contente parfois de simples analogies avec la photographie ou le cinéma : "Recuerdo aquel lunes como un conjunto de escenas de una película cuyo actor era yo. " (El mundo, 200). Le passé remonte ainsi à la surface comme des "secuencias inconexas, como pedazos de pelicula rescatados de un rollo roto" (El mundo, 16), évidemment "en blanco y negro" (17). À d'autres moments, le texte est littéralement mis en scène. Comme dans ces pages où le narrateur livre à l'état brut quelques plans séquences, sorte de rushs avant montage ( $E l$ mundo, 16). Le procédé ici s'ajuste parfaitement au réel que cherche à signifier le narrateur puisque le faire redouble le dire. La phrase qui introduit ces plans séquences est en effet celle-ci : "todo estaba roto" (El mundo, 16), phrase récurrente et fondatrice des romans de Millás ${ }^{20}$.

Tout au long du roman, les exemples de ce type d'artifice permettant de découper le réel, de produire des effets de cadrage se multiplient. Les figures médiatrices peuvent être des plus diverses, ainsi, par exemple, la « ventanilla del tranvía" (p. 54) ou encore la fenêtre de la salle de classe produisent un effet de cadrage depuis lequel le point de vue est altéré et le monde devient autre. Parmi les motifs s'inscrivant dans ces procédés, le miroir, qui "enajena a la vez que establece coincidencia ", est sans doute l'objet symbolisant à lui seul la dualité de l'écran obstacle et de l'écran réceptacle ${ }^{21}$.

Du cadre à l'écran, la frontière est mince, d'ailleurs les deux sont souvent mêlés dans le texte. Est écran tout ce qui vient interférer entre le narrateur personnage, Juanjo, et le monde qui l'entoure. Mais faire écran, ce n'est pas rendre impossible la saisie de l'objet regardé, c'est permettre que filtre et s'affiche une autre dimension d'un réel qui se dérobe. Dans toute l'œuvre de Millás, on trouve des exemples de cet effet-écran qui s'incarne dans une multiplicité de motifs ou de figures. Les états frontières que sont la fièvre, le songe ou le rêve sont autant d'écrans entre le protagoniste et le réel qui permettent à celui-là de prendre la mesure de celui-ci: "Recuerdo todas y cada una de las ocasiones en las que he visto el mundo a través de la fiebre. " (El mundo, 65), "[...] la fiebre crea una red de dolor dulce que te conecta a la realidad, al mundo, a la tierra..." (El mundo, 66). Les barbituriques aussi ont cette capacité : " a los diez minutos se instalaba entre la realidad y yo una suerte de nebulosa que facilitaba nuestra relación" (El mundo, 27)

Écran ultime sur lequel s'affiche l'ensemble du processus d'écriture du roman, le narrateur devient un passeur, une instance blanche permettant que le monde advienne : "Comprendí que yo, finalmente, no era más que un escenario en el que habia ocurrido cuanto se relataba en El mundo. " (El mundo, 232).

20. On pourrait encore citer l'exemple du jeu sur le positif et le négatif, deux versants d'une même réalité, lorsque le narrateur évoque un souvenir d'enfance à Valencia : il croisait souvent un enfant aveugle qui habitait le quartier, et il s'imaginait qu'il lui suffisait de fermer les yeux, c'est-à-dire qu'il devienne lui-même aveugle, pour que l'enfant aveugle retrouve la vue l'espace de cet instant. (El mundo, 19)

21. On sait que chez Millás le miroir est chargé de sens, en particulier comme motif essentiel du thème littéraire du double, mais aussi comme objet et symbole structurant de la psychanalyse. 
Mais derrière ce narrateur-écran perce ce qui est sans doute le médiateur le plus essentiel, et à la fois le plus manifeste et évident : le langage. Un langage que le jeune Juanjo doit apprivoiser, décoder, s'approprier, car il est la matièremême à partir de laquelle la référence peut s'exercer. Ainsi, dans une tension autoréférentielle constante et depuis la conscience de faire livre (le titre apparaît à la page 233), El mundo s'avère être aussi une réflexion profonde sur l'écriture et sa capacité à générer du réel. Ce qui explique que de nombreuses phrases émaillent le texte, constituant un fil métadiscursif toujours tourné vers la diction du monde : "La lectura y la escritura son también espacios desde los que no siempre, pero de vez en cuando, se ve la calle, quiero decir la Calle, o sea, el mundo." (El mundo, 105). Dans une longue digression réflexive qui prend des airs de columna insérée, le narrateur joue sur/avec les mots et, après quelques détours humoristiques sur la matière du langage et l'arbitraire ou la juste motivation du signe linguistique, le narrateur est amené à conclure qu'il assume ce rôle de réparateur de réalité par le langage, grâce à ces outils que sont les mots : "Arreglar la realidad resultaba agotador, pero alguien tenía que ocuparse de ello" (El mundo, 69)

\section{DE LA LANGUE ÉCRAN À LA LANGUE ÉCRIN}

Marqué, à vie semble-t-il, par cette phrase définitive que María José, dont il était amoureux, lui avait opposée : "Tú no eres interesante para mí." (141), le narrateur y voit a posteriori un moment fondateur de l'écrivain qu'il est devenu. Retournée dans tous les sens, la phrase montre a quel point la réalité est dépendante du langage, car par un simple écart, une petite virgule qui vient faire écran à la réalité douloureuse, l'écriture permet à l'écrivain de conjurer la souffrance:

Estar muerto era en mi situación un consuelo, pues cómo soportar vivo, no ya aquel rechazo, sino aquella humillación. Tú no eres interesante para mí. En una de las miles de veces que repetí la frase, reconstruyendo la situación para ver si le encontraba una salida, pensé que entre el "tú no eres interesante» y el "para mì habia habido una pequeña pausa, una cesura, que dejaba una vía de escape. Quizá habia dicho: "Tú no eres interesante, para mi.» La coma entre el "interesante» y el "para» venía a significar que podía ser interesante para otros, incluso para el mundo en general. Era la primera vez que le encontraba utilidad práctica a un signo ortográfico, la primera vez que le encontraba un sentido a la gramática. Quizá al colocar aquella coma perpetré un acto fundacional, quizá me hice escritor en ese instante. Tal vez descubrimos la literatura en el mismo acto de fallecer. (El mundo, 141-

L'effet-écran est aussi à l'œuvre dans les jeux avec les temps. Le bilan de son existence que fait le narrateur s'actualise dans des allers-retours incessants entre passé et présent de l'énonciation entre lesquels vient s'immiscer un temps intermédiaire, un temps écran : le présent de narration, qui certes donne de la vivacité au récit, mais marque aussi l'incidence du passé sur le présent, fond les deux et signifie la trace de l'un dans l'autre. 
El mundo est tout à la fois une reconstruction de la trajectoire d'un auteur conscient d'avoir élaboré une œuvre cousue à une réalité, la sienne, faite d'expériences personnelles sans cesse remises en perspective. Toutes ces expériences semblent pourtant ne converger que vers un but: la nécessaire conquête du langage et de l'écriture. L'écriture est le médiateur le plus puissant pour dévoiler le monde, ce qui ne signifie pas chez Millás réduire la littérature à une simple chronique. Au contraire il s'agit bien de référer au monde préexistant tout en créant son propre référent depuis la conscience même de l'écriture, d'une écriture jamais acquise, toujours à construire. Tout comme le jeune Millás pouvait voir les filtrations du monde à travers la grille crasseuse du fenestron, le réel vient s'immiscer dans le texte par toute une série de détours, autant de procédés médiateurs créant un puissant réseau métatextuel : la référence et l'autoréférence se superposent, s'éclairent mutuellement, non plus comme deux pôles contraires ou complémentaires d'une même tension, mais comme la seule unité possible dans l'ordre du roman. Ainsi, par analogie, les frappes sur le clavier de l'ordinateur sont des échos des gouttes d'eau de ce lundi cruel de l'enfance où le narrateur fuit la violence du directeur de l'école où ses parents l'avaient inscrit pour une remise à niveau $(E l$ mundo, 202). Ou encore par exsudation, comme l'affirme le narrateur lui-même : " a veces en las novelas se filtran fragmentos de realidad que dejan manchas de humedad, como una gotera en la pared de una habitación." (El mundo, 136).

Le monde du jeune protagoniste de El mundo, écrivain en devenir, est encore celui de l'affect et non du discours. Ce que retrace le roman, c'est bien la conquête du discours. C'est ce que laissent entendre les allusions récurrentes aux actes fondateurs de l'écriture. Comme à son habitude, Millás avance à visage découvert, dénudant les procédés parfois complexes qu'il met du temps à construire, et dévoile dans de fréquents passages métadiscursifs, le faire même du texte. L'enjeu est donné dès les premières pages. La phrase fondatrice du roman est répétée, voire assenée, à plusieurs reprises, notamment aux deux extrémités du texte :

Cuando escribo a mano, sobre un cuaderno, como ahora, creo que me parezco un poco a mi padre en el acto de probar el bisturí eléctrico, pues la escritura abre y cauteriza al mismo tiempo las heridas ${ }^{22}$. (El mundo, 8)

Volvi a escuchar la frase fundacional de esta novela, quizá del resto de mi vida (cauteriza la herida al tiempo de abrirla) (El mundo, 225)

Lécriture est réparatrice, le constat n'est certes pas très original, mais elle est surtout fondatrice et constitutive : " ...mi padre se relacionaba con sus herramientas como si fueran prolongaciones de su cuerpo, un conjunto de protesis. Del mismo modo que el lenguaje nos utiliza y nos moldea hasta el punto de que, más que hablar con él, somos hablados por él, mi padre parecía hablado por las herramientas que tenía siempre a mano." (El mundo, 25).

22. C’est moi qui souligne. 
À la fois écran et écrin, elle est ce par quoi le monde et El mundo adviennent. Ainsi, lorsque le narrateur part à Valencia disperser en mer les cendres de ses parents, comme la métaphore d'un réel dissous, celles-ci se matérialisent dans l'écriture à travers une analogie mettant en abyme l'écriture même du texte. C'est la fin du roman et les cendres se font texte : "Así logré que le mar se llevara una parte sustancial [de las cenizas], pero la otra se quedó como impresa en la superficie de la arena, formando dibujos que parecian un alfabeto." (El mundo, 227).

\section{Conclusion}

El mundo est un texte emblématique des rapports référence/autoréférence dans la mesure où le narrateur y fait dialoguer deux strates : son rapport au monde familial, social et finalement historique, et son rapport à l'écriture. Audelà de ce dialogue, ce que nous propose Millás est une réflexion sur la capacité même de la langue à dire le monde, sur la nécessité de trouver des stratagèmes, des procédures, par lesquelles parvenir à en rendre compte. Car si, comme il semble le dire à longueur de columna, l'accès au réel est très problématique dans le réel-même, il l'est a fortiori dans le texte littéraire. Ainsi face à son impossible saisie immédiate, Millás l'envisage et l'appréhende de façon médiate, c'està-dire à travers des figures médiatrices qui tout à la fois le déforment et en assurent la représentation Nous retrouvons là les deux obsessions fondatrices de l'œuvre de Millás : le réel et l'écriture.

C'est donc en se disant que le texte dit le monde. En mettant l'accent sur le discours qui sous-tend la référence, c'est-à-dire en travaillant la tension autoréférentielle, le texte de Millás ne fait qu’affirmer la qualité transitive de son écriture fictionnelle, capable d'engendrer à la fois l'histoire d'un récit et le récit d'une histoire. Il s'agit encore et toujours chez lui de «dar cuento de la realidad $^{23}$ ».

23. La belle formule est empruntée à Albert Chillón, qui l'applique aux articles littéraires de Juan José Millás. Albert Chillón, Literatura y periodismo. Una tradición de relaciones promiscuas, Barcelone, Aldea Global, 1999. 